\title{
hudson: A User-Friendly R Package to Extend Manhattan Plots
}

\author{
Anastasia Lucas ${ }^{1}$, Anurag Verma ${ }^{1,2,3}$, Marylyn D. Ritchie ${ }^{1,3}$ \\ ${ }^{1}$ Department of Genetics, Perelman School of Medicine, University of Pennsylvania, Philadelphia, \\ PA, United States \\ ${ }^{2}$ Institute for Biomedical Informatics, Perelman School of Medicine, University of Pennsylvania, \\ Philadelphia, PA, United States \\ ${ }^{3}$ Department of Medicine, Perelman School of Medicine, University of Pennsylvania, Philadelphia, \\ PA, United States
}

\author{
* Correspondence: \\ Marylyn D. Ritchie \\ marylyn@pennmedicine.upenn.edu
}

\section{Keywords: visualization, genomics, multivariate data, GWAS, PheWAS}

\begin{abstract}
The Manhattan plot is one of the most widely used visualization techniques when plotting summary statistics from genome-wide or phenome-wide association studies. While there are a number of existing tools to create these plots, there is room for extending their utility to satisfy increasingly complex and comprehensive analyses as well as the need for comparisons between different sets of results or between discovery and replication datasets. The $\mathrm{R}$ package presented here, hudson, provides user-friendly plotting functions intended for use with genome, phenome, and exposomewide association studies, but its flexible framework can be utilized for a wide variety genome-wide analyses. Further, we have extended these figures to allow for interactive elements to facilitate results exploration for the ever-increasing large-scale dimensionality of these data. hudson can be obtained from https://github.com/RitchieLab/hudson.
\end{abstract}

\section{Introduction}

Manhattan plots displaying p-values at a genome scale have long been considered the standard method to provide a big-picture overview of genome-wide association study (GWAS) results. As genome- ${ }^{1}$, phenome- ${ }^{2}$, and exposome ${ }^{3}$ wide analyses (GWAS, PheWAS, EWAS) become more sophisticated and involve more dimensions of data, there is room to further develop and improve on data visualization techniques for the presentation of results. Including post-GWAS analysis methods and study designs that extend beyond basic GWAS, such as comparisons with previously published summary statistics, sex-stratified analyses, gene-based studies, transcriptome-wide association studies, etc., is becoming the norm in these large-scale scientific studies of the genetic or genomic architecture of complex traits. To perform comparisons of different datasets or different sets of results, using popular Manhattan plot packages such as qqman ${ }^{4}$ or PheWAS $^{5}$ would require separate or side-by-side figures presented for comparison; these can be difficult to mentally map the 
chromosome positions, a task necessary to notice key similarities and differences in loci. It can also be challenging to drill down to specific results as most of these packages produce static images that make it difficult to discern specific data points.

While there exist several packages such as miamiplot ${ }^{6}$, gwaRs $^{7}$, and karyoploteR ${ }^{8}$, for comparing two Manhattan plots using SNP data from association studies, many are inflexible to data that deviates from a traditional GWAS study and are constrained to a static figure that is limited to three or four attributes - $\mathrm{x}$-axis position, $\mathrm{y}$-axis position, color, and/or shape. To the authors' knowledge, there are no maintained Manhattan plot packages that include methods for creating mirrored Manhattan plot figures as well as allow for data that cannot be organized into chromosomes, such as exposures or tissues, and include methods to create interactive figures that allow the user to view meta data without the need for heavy coding. Here, we present hudson, an R package to create mirrored Manhattan plots for various data types with added capabilities to create interactive figures with the goal of providing a single easy to use data visualization resource for researchers.

\section{Methods}

hudson was developed as an open-source $\mathrm{R}$ package. The plotting mechanisms are dependent on the widely used graphing library ggplot $2^{9}$ and the interactive functions utilize the interactive ggplot extension, ggiraph ${ }^{10}$. Additionally, hudson will make use of ggrepel ${ }^{11}$, an $\mathrm{R}$ library that automatically repels data labels to prevent overlapping text, if it is installed on the user's system. The package consists of seven main functions, two each designed for both static and interactive visualization of GWAS, PheWAS, and EWAS analysis comparisons and also a QQ-plot function. Sometimes referred to as Miami plots ${ }^{12,13}$ or Chicago plots ${ }^{14}$ our Hudson plots are characterized by a divergent yaxis and a shared $\mathrm{x}$-axis, allowing for a position by position, or more generally, variable by variable, comparison of data.

In their most basic form, each half of the Hudson plot consists of a typical Manhattan or PheWAS Manhattan plot. The x-axis generally represents either 1) chromosome and base pair location across the genome or 2) phenotype/disease categories. From there, users can choose to set multiple parameters to highlight and/or annotate above certain p-value thresholds or by name, add threshold lines, and make various theme changes such as changing the background or colors, turning on or off $\mathrm{x}$-axis marker blocks, and manually adjusting the y-axes. The functions designed to work with genomic data can also accept any number of chromosomes such that the user can plot non-human genomes or any subset of interest such as specific gene sets as shown in Drivas et al $2021^{15}$.

Users wishing to make interactive versions of figures provide custom annotations in additional metadata columns. This metadata will be displayed when the user hovers over a point on the figure. This allows a user to interact with the data and for each point determine the characteristics of that data point (for example, SNP ID, gene ID, odds ratio or beta, and p-value). Further, the user can provide a link that should be opened in a browser window when the user clicks on the point; this could link out to the dbSNP information about that variant, OMIM information about that gene, or a publication in PubMed for example. These features eliminate the need to perform manual lookups when investigating SNPs or genes of interest. The interactive figures can then be saved as HTML files which can easily be shared among researchers and opened locally in any internet browser.

As one of the goals of the package is to produce publication quality images, we have considered the need for color-blind friendly color palettes. By default, hudson will select colors from a 15-color palette that is distinguishable for people with deuteranopia and protanopia, and generally safe for 
tritanopia ${ }^{*}$. If more than 15 colors are required, hudson will interpolate colors from Google AI's Turbo palette ${ }^{\dagger}$ which is both perceptually uniform and distinguishable for people with all forms of partial colorblindness, offering a benefit over a traditional rainbow palette. Although users will be able to override these palettes and specify their own color scheme, we hope that having them built in as defaults will make it easier for users to create more widely accessible figures.

Though we have provided a flexible framework for visualizing many types of data, we recognize that there will be instances where the user requires further customization or features and, therefore, have made the source code for both packages freely available on GitHub under the GPL-3 license. Additionally, for the most part, each function is able to be downloaded, modified if needed, and run as a standalone script in R. For example, in Veturi et al. 2021, we modified hudson code to generate Figure 3 in which we rotated the plot 90 degrees so that we could display two Hudson plots side by side to compare gene expression based PheWAS (Xpress-PheWAS) and SNP-based PheWAS in two datasets ${ }^{16}$. The package was designed this way intentionally to increase accessibility to researchers who wish to make small tweaks to their figures, but do not have as much experience with R package development.

\section{Usage}

Figure 1 provides a more comprehensive example of the a few of the use cases for the hudson package using summary statistics obtained from the NHGRI-EBI GWAS Catalog ${ }^{17}$ and the default data from the hudson package. Panel A displays standard Hudson plot with GWAS results from the Ehret et al. blood pressure $\mathrm{GWAS}^{18}$ for two traits, systolic blood pressure (SBP) and diastolic blood pressure (DBP). Panel B displays all four phenotypes from the Spracklen et al. lipid GWAS results ${ }^{19}$ along with the betas for $\mathrm{X}$ in the top and $\mathrm{Y}$ in the bottom. Panel $\mathrm{C}$ displays an example of an exposome-wide association study using a dummy dataset provided by the hudson package. Panel D displays a gene-based PheWAS plot. These examples were selected to demonstrate the wide variability of types of data that can be displayed in Hudson plots.

Figure 2 shows an example of an interactive PheWAS Hudson plot figure. This plot includes the data from the Spracklen et al paper. The methods used to create the individual panels for each of these figures are explained in more detail below and the code used to create the figures can be found at https://github.com/RitchieLab/utility/tree/master/personal/ana/hudson-paper. 


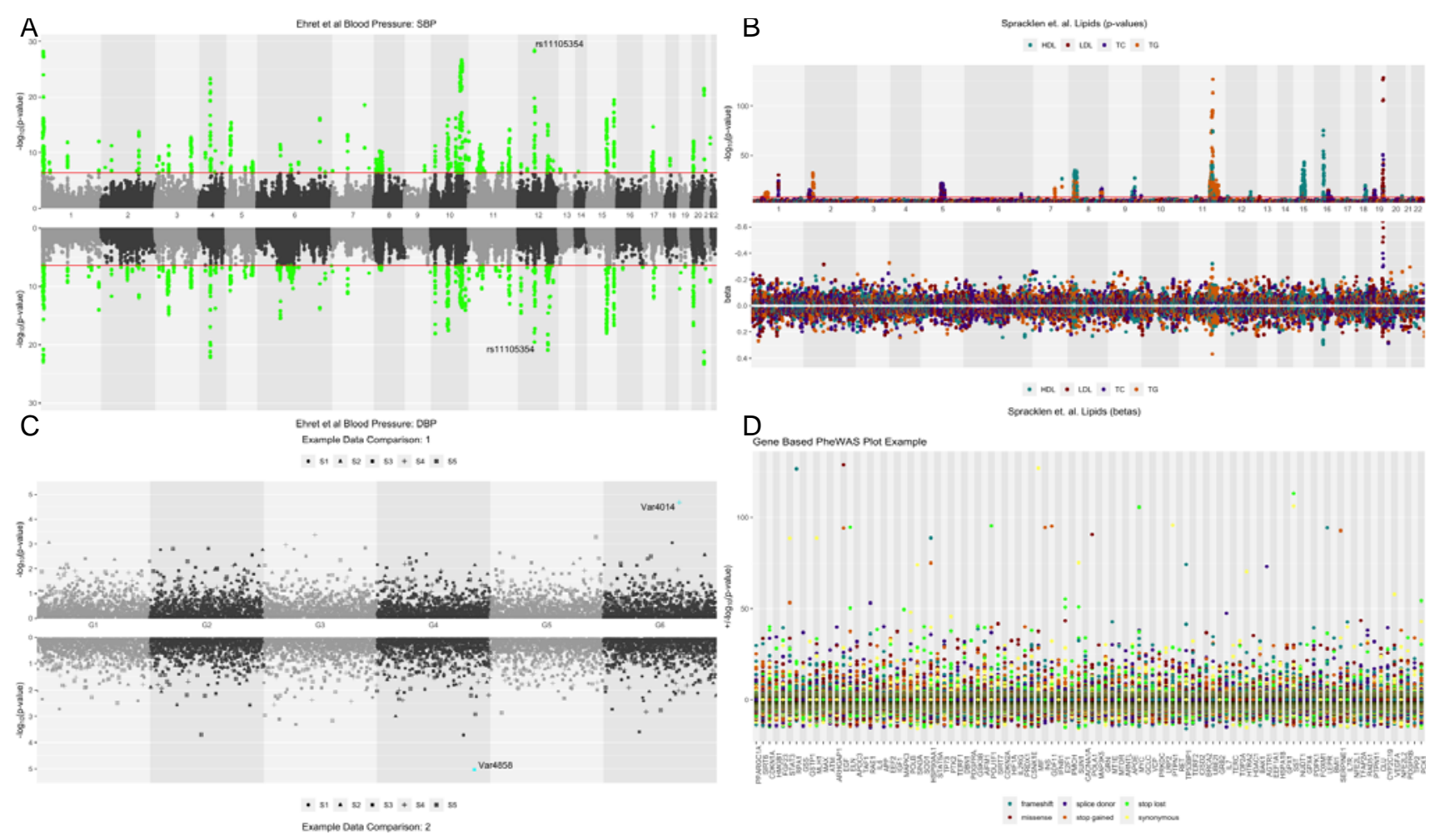

Figure 1: Examples of GWAS (A), PheWAS (B), and EWAS (C) hudson plots, each showing different use cases for the hudson package. Panel D shows an example of a modified version of the PheWAS plotting method that organizes variants by gene in lieu of chromosomes. Note the complete lack of linkage disequilibrium characteristic of fabricated data in comparison with Figures 2-5 of Drivas, et al.
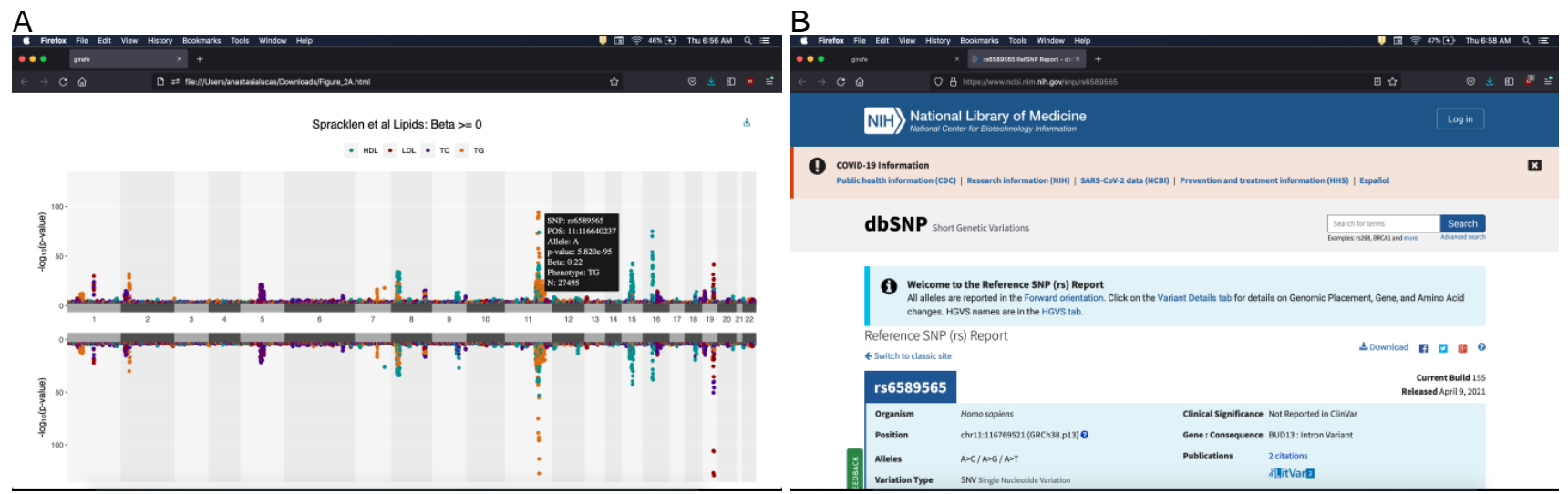

Figure 2: Screenshot of an interactive PheWAS Hudson plot being viewed in an internet browser. Panel A shows an example of annotations that will appear when a point corresponding to a SNP is hovered over while panel B shows the dbSNP entry for that variant opening in a new browser window when the user clicks on the point.

\subsection{Mirrored GWAS plots}

GWAS Hudson plots produced by the gmirror() function will accept as input genomic data such as those output from common GWAS analysis tools like PLINK ${ }^{20}$. Thus, data should contain at least columns for variant name, chromosome, position, and p-value (or other numeric value, such as beta). As in the other plotting functions, an additional layer of information can optionally be included in 
column mapped to shape. Figure 1A contains an example of a GWAS Hudson plot using summary statistics from the GWAS Catalog for studies GCST006258 ${ }^{18}$ and GCST006259 ${ }^{18}$ downloaded on $11 / 22 / 2021$. This example makes use of hudson's p-value threshold highlighting and variant annotation options.

\subsection{Mirrored PheWAS plots}

PheWAS Hudson plots are produced by the phemirror() function and take input genomic data similar to described above, just with the addition of a phenotype column that will be automatically mapped to a color. Note that this "phenotype" could be any attribute the user wishes to map to color, for example variant annotations, such that this is not solely limited to visualizing data from a PheWAS analysis. Figure 1B shows an example of visualizing two different data types from a collection of four traits from a lipid association study ${ }^{19}$ (GCST004232, GCST004233, GCST004235 and GCST004237) downloaded from the GWAS Catalog on 10/24/2021. Here we see p-values in the upper panel and beta in the lower panel, making use of hudson's free y-axis feature. This panel also shows the use of chromosome panels and base blocks to better distinguish which points belong to which chromosome.

\subsection{Non-genomic and other plot types}

Although we have referred to the types of figures the emirror() function can create as EWAS plots, this function is suitable for any analysis where many results are organized into categories, such as tissues or pathways, provided there is a common $\mathrm{x}$-axis between the upper and lower panels. Thus, only three columns of information are needed: variant, group, and p-value or other numeric value. Figure $1 \mathrm{C}$ is an example of a plot using the simulated EWAS data provided by the package with an additional attribute mapped to shape. Here, we again can use similar variable (and/or p-value) highlighting thresholds and theme options as described above.

To highlight the flexibility of the package, we have also included an example that is not made by the default package, but was obtained with only minor modifications to the source code in Figure 1D. This version of the mirrored PheWAS plot uses gene names as our pseudo 'chromosome' grouping, achieved with the addition of a single parameter to specify ordering, and was further modified to use a fixed position for the variants rather than a number line. The code used to create this figure can be found at https://github.com/RitchieLab/utility/blob/master/personal/ana/scripts/phegene_hudson.R.

\subsection{Interactive plots}

Users wishing to make interactive versions of the figures can provide data in the same input format described above to analogous functions prefixed with ' $\mathrm{i}$ ', i.e. igmirror(), iphemirror(), and iemirror(). However, here they may optionally provide custom annotations in an additional metadata column. This metadata will be displayed when the user hovers over a point on the figure as shown in the screenshot of an interactive PheWAS Hudson plot being viewed in an internet browser in Figure $2 \mathrm{~A}$. Various user-defined annotations including the variant name, chromosomal position, beta, effect allele, and number of samples can be added. Further, the user can provide a link that should be opened in a new browser window when the user clicks on the point, as demonstrated in Figure $2 \mathrm{~B}$. This link could be anything from a dbSNP query to a Google search. Both the annotations and connections to external databases eliminate, or at least greatly reduce, the need to perform manual lookups when initially investigating SNPs, genes, or other variables of interest. The interactive figures are then saved as HTML files which can easily be shared among researchers and can be opened in any internet browser. 


\section{Discussion}

The hudson package allows users to easily create publication quality or shareable interactive mirrored Manhattan plot figures. When performing association analyses on hundreds of thousands or even millions of variables, it can be a challenge to interpret the results from large tables alone. Visualizing the results in different ways and specifically with the ability to compare different groups in a single plot with accurate alignment of the variables can make this interpretation process significantly more accurate and efficient. In hudson, we provide a flexible, open-source framework that allows users to visualize data from a wide variety of study designs as well as annotate those figures with interactive metadata, while also encouraging more advanced users to customize the scripts to meet individual research needs that are not explicitly fulfilled by the base package.

\section{Conflict of Interest}

The authors declare that the research was conducted in the absence of any commercial or financial relationships that could be construed as a potential conflict of interest.

\section{Author Contributions}

AL conceived of and developed the software package and wrote the manuscript. AV made design contributions to the package. MDR supervised the project. All authors contributed to editing the article and approved the submitted version.

\section{Funding}

This work was supported in part by GM115318 and AI077505.

\section{Acknowledgments}

We acknowledge GitHub user @ dbaranger who published R code for the Turbo color palette implemented in the package.

\section{Data Availability Statement}

Example datasets used in this study can be found in the GWAS Catalog (https://www.ebi.ac.uk/gwas/ and toy data can be found at Ritchie Lab GitHub (https://github.com/RitchieLab/hudson/tree/master/data) or loaded from the package itself.

\section{References}

1. Alder JK, Kass DJ. Another building in the IPF Manhattan plot skyline. Lancet Respir Med. 2017 Nov;5(11):837-839. doi: 10.1016/S2213-2600(17)30394-6. Epub 2017 Oct 20. PMID: 29066087.

2. Verma A, Lucas A, Verma SS, Zhang Y, Josyula N, Khan A, Hartzel DN, Lavage DR, Leader J, Ritchie MD, Pendergrass SA. PheWAS and Beyond: The Landscape of Associations with Medical Diagnoses and Clinical Measures across 38,662 Individuals from Geisinger. Am J Hum Genet. 2018 Apr 5;102(4):592-608. doi: 10.1016/j.ajhg.2018.02.017. Epub 2018 Mar 29. PMID: 29606303; PMCID: PMC5985339. 
3. Chung MK, Buck Louis GM, Kannan K, Patel CJ. Exposome-wide association study of semen quality: Systematic discovery of endocrine disrupting chemical biomarkers in fertility require large sample sizes. Environ Int. 2019;125:505-514. doi:10.1016/j.envint.2018.11.037

4. Turner, (2018). qqman: an R package for visualizing GWAS results using Q-Q and manhattan plots. Journal of Open Source Software, 3(25), 731, https://doi.org/10.21105/joss.00731

5. Carroll RJ, Bastarache L, Denny JC. R PheWAS: data analysis and plotting tools for phenomewide association studies in the R environment. Bioinformatics. 2014 Aug 15;30(16):2375-6. doi: 10.1093/bioinformatics/btu197. Epub 2014 Apr 14. PMID: 24733291; PMCID: PMC4133579.

6. White, Julie. (2020). ggraph: Create a ggplot2 miami plot. R package version 1.1.0. Available online at: https://github.com/juliedwhite/miamiplot

7. Nkambule LL. gwaRs: an R shiny web application for visualizing genome-wide association studies data. bioRxiv [Preprint]. Available at: https://doi.org/10.1101/2020.04.17.044784 (Accessed October 6th, 2021)

8. Bernat Gel, Eduard Serra, karyoploteR: an R/Bioconductor package to plot customizable genomes displaying arbitrary data, Bioinformatics, Volume 33, Issue 19, 01 October 2017, Pages 3088-3090, https://doi.org/10.1093/bioinformatics/btx346

9. Wickham, H. (2016). ggplot2: Elegant Graphics for Data Analysis. Springer-Verlag New York. Retrieved from https://ggplot2.tidyverse.org

10. Gohel D, Skintzos P. (2021). Create interactive 'ggplot2' graphics using 'htmlwidgets'. R package version 0.7.10. Available online at: https://CRAN.R-project.org/package=ggiraph

11. Kamil Slowikowski (2021). ggrepel: Automatically Position Non-Overlapping Text Labels with 'ggplot2'. R package version 0.9.1. https://CRAN.R-project.org/package=ggrepel

12. Winkler TW, Kutalik Z, Gorski M, Lottaz C, Kronenberg F, Heid IM. EasyStrata: evaluation and visualization of stratified genome-wide association meta-analysis data. Bioinformatics. 2015;31(2):259-261. doi:10.1093/bioinformatics/btu621

13. Gibson J, Russ TC, Clarke TK, Howard DM, Hillary RF, Evans KL, Walker RM, Bermingham ML, Morris SW, Campbell A, Hayward C, Murray AD, Porteous DJ, Horvath S, Lu AT, McIntosh AM, Whalley HC, Marioni RE. A meta-analysis of genome-wide association studies of epigenetic age acceleration. PLoS Genet. 2019 Nov 18;15(11):e1008104. doi:

10.1371/journal.pgen.1008104. PMID: 31738745; PMCID: PMC6886870.

14. Choquet H, Melles RB, Anand D, et al. A large multiethnic GWAS meta-analysis of cataract identifies new risk loci and sex-specific effects. Nat Commun. 2021;12(1):3595. Published 2021 Jun 14. doi:10.1038/s41467-021-23873-8

15. Drivas TG, Lucas A, Zhang X, Ritchie MD. Mendelian pathway analysis of laboratory traits reveals distinct roles for ciliary subcompartments in common disease pathogenesis. Am J Hum Genet. 2021;108(3):482-501. doi:10.1016/j.ajhg.2021.02.008

16. Veturi Y, Lucas A, Bradford Y, Hui D, Dudek S, Theusch E, Verma A, Miller JE, Kullo I, Hakonarson H, Sleiman P, Schaid D, Stein CM, Edwards DRV, Feng Q, Wei WQ, Medina MW, Krauss RM, Hoffmann TJ, Risch N, Voight BF, Rader DJ, Ritchie MD. A unified framework identifies new links between plasma lipids and diseases from electronic medical records across large-scale cohorts. Nat Genet. 2021 Jul;53(7):972-981. doi: 10.1038/s41588021-00879-y. Epub 2021 Jun 17. PMID: 34140684; PMCID: PMC8555954.

17. Buniello A, MacArthur JAL, Cerezo M, Harris LW, Hayhurst J, Malangone C, McMahon A, Morales J, Mountjoy E, Sollis E, Suveges D, Vrousgou O, Whetzel PL, Amode R, Guillen JA, Riat HS, Trevanion SJ, Hall P, Junkins H, Flicek P, Burdett T, Hindorff LA, Cunningham F and Parkinson H. The NHGRI-EBI GWAS Catalog of published genome-wide association studies, targeted arrays and summary statistics 2019. Nucleic Acids Research, 2019, Vol. 47 (Database issue): D1005-D1012. 
18. Ehret GB, Ferreira T, Chasman DI, Jackson AU, Schmidt EM, Johnson T, Thorleifsson G, Luan J, Donnelly LA, Kanoni S, Petersen AK, Pihur V, Strawbridge RJ, Shungin D, Hughes MF, Meirelles O, Kaakinen M, Bouatia-Naji N, Kristiansson K, Shah S, Kleber ME, Guo X, Lyytikäinen LP, Fava C, Eriksson N, Nolte IM, Magnusson PK, Salfati EL, Rallidis LS, Theusch E, Smith AJP, Folkersen L, Witkowska K, Pers TH, Joehanes R, Kim SK, Lataniotis L, Jansen R, Johnson AD, Warren H, Kim YJ, Zhao W, Wu Y, Tayo BO, Bochud M; CHARGE-EchoGen consortium; CHARGE-HF consortium; Wellcome Trust Case Control Consortium, Absher D, Adair LS, Amin N, Arking DE, Axelsson T, Baldassarre D, Balkau B, Bandinelli S, Barnes MR, Barroso I, Bevan S, Bis JC, Bjornsdottir G, Boehnke M, Boerwinkle E, Bonnycastle LL, Boomsma DI, Bornstein SR, Brown MJ, Burnier M, Cabrera CP, Chambers JC, Chang IS, Cheng CY, Chines PS, Chung RH, Collins FS, Connell JM, Döring A, Dallongeville J, Danesh J, de Faire U, Delgado G, Dominiczak AF, Doney ASF, Drenos F, Edkins S, Eicher JD, Elosua R, Enroth S, Erdmann J, Eriksson P, Esko T, Evangelou E, Evans A, Fall T, Farrall M, Felix JF, Ferrières J, Ferrucci L, Fornage M, Forrester T, Franceschini N, Duran OHF, Franco-Cereceda A, Fraser RM, Ganesh SK, Gao H, Gertow K, Gianfagna F, Gigante B, Giulianini F, Goel A, Goodall AH, Goodarzi MO, Gorski M, Gräßler J, Groves C, Gudnason V, Gyllensten U, Hallmans G, Hartikainen AL, Hassinen M, Havulinna AS, Hayward C, Hercberg S, Herzig KH, Hicks AA, Hingorani AD, Hirschhorn JN, Hofman A, Holmen J, Holmen OL, Hottenga JJ, Howard P, Hsiung CA, Hunt SC, Ikram MA, Illig T, Iribarren C, Jensen RA, Kähönen M, Kang H, Kathiresan S, Keating BJ, Khaw KT, Kim YK, Kim E, Kivimaki M, Klopp N, Kolovou G, Komulainen P, Kooner JS, Kosova G, Krauss RM, Kuh D, Kutalik Z, Kuusisto J, Kvaløy K, Lakka TA, Lee NR, Lee IT, Lee WJ, Levy D, Li X, Liang KW, Lin H, Lin L, Lindström J, Lobbens S, Männistö S, Müller G, Müller-Nurasyid M, Mach F, Markus HS, Marouli E, McCarthy MI, McKenzie CA, Meneton P, Menni C, Metspalu A, Mijatovic V, Moilanen L, Montasser ME, Morris AD, Morrison AC, Mulas A, Nagaraja R, Narisu N, Nikus K, O'Donnell CJ, O'Reilly PF, Ong KK, Paccaud F, Palmer CD, Parsa A, Pedersen NL, Penninx BW, Perola M, Peters A, Poulter N, Pramstaller PP, Psaty BM, Quertermous T, Rao DC, Rasheed A, Rayner NWNWR, Renström F, Rettig R, Rice KM, Roberts R, Rose LM, Rossouw J, Samani NJ, Sanna S, Saramies J, Schunkert H, Sebert S, Sheu WH, Shin YA, Sim X, Smit JH, Smith AV, Sosa MX, Spector TD, Stančáková A, Stanton A, Stirrups KE, Stringham HM, Sundstrom J, Swift AJ, Syvänen AC, Tai ES, Tanaka T, Tarasov KV, Teumer A, Thorsteinsdottir U, Tobin MD, Tremoli E, Uitterlinden AG, Uusitupa M, Vaez A, Vaidya D, van Duijn CM, van Iperen EPA, Vasan RS, Verwoert GC, Virtamo J, Vitart V, Voight BF, Vollenweider P, Wagner A, Wain LV, Wareham NJ, Watkins H, Weder AB, Westra HJ, Wilks R, Wilsgaard T, Wilson JF, Wong TY, Yang TP, Yao J, Yengo L, Zhang W, Zhao JH, Zhu X, Bovet P, Cooper RS, Mohlke KL, Saleheen D, Lee JY, Elliott P, Gierman HJ, Willer CJ, Franke L, Hovingh GK, Taylor KD, Dedoussis G, Sever P, Wong A, Lind L, Assimes TL, Njølstad I, Schwarz PE, Langenberg C, Snieder H, Caulfield MJ, Melander O, Laakso M, Saltevo J, Rauramaa R, Tuomilehto J, Ingelsson E, Lehtimäki T, Hveem K, Palmas W, März W, Kumari M, Salomaa V, Chen YI, Rotter JI, Froguel P, Jarvelin MR, Lakatta EG, Kuulasmaa K, Franks PW, Hamsten A, Wichmann HE, Palmer CNA, Stefansson K, Ridker PM, Loos RJF, Chakravarti A, Deloukas P, Morris AP, Newton-Cheh C, Munroe PB. The genetics of blood pressure regulation and its target organs from association studies in 342,415 individuals. Nat Genet. 2016 Oct;48(10):1171-1184. doi: 10.1038/ng.3667. Epub 2016 Sep 12. PMID: 27618452; PMCID: PMC5042863.

19. Spracklen CN, Chen P, Kim YJ, Wang X, Cai H, Li S, Long J, Wu Y, Wang YX, Takeuchi F, Wu JY, Jung KJ, Hu C, Akiyama K, Zhang Y, Moon S, Johnson TA, Li H, Dorajoo R, He M, Cannon ME, Roman TS, Salfati E, Lin KH, Guo X, Sheu WHH, Absher D, Adair LS, Assimes TL, Aung T, Cai Q, Chang LC, Chen CH, Chien LH, Chuang LM, Chuang SC, Du S, Fan Q, 
Fann CSJ, Feranil AB, Friedlander Y, Gordon-Larsen P, Gu D, Gui L, Guo Z, Heng CK, Hixson J, Hou X, Hsiung CA, Hu Y, Hwang MY, Hwu CM, Isono M, Juang JJ, Khor CC, Kim YK, Koh WP, Kubo M, Lee IT, Lee SJ, Lee WJ, Liang KW, Lim B, Lim SH, Liu J, Nabika T, Pan WH, Peng H, Quertermous T, Sabanayagam C, Sandow K, Shi J, Sun L, Tan PC, Tan SP, Taylor KD, Teo YY, Toh SA, Tsunoda T, van Dam RM, Wang A, Wang F, Wang J, Wei WB, Xiang YB, Yao J, Yuan JM, Zhang R, Zhao W, Chen YI, Rich SS, Rotter JI, Wang TD, Wu T, Lin X, Han BG, Tanaka T, Cho YS, Katsuya T, Jia W, Jee SH, Chen YT, Kato N, Jonas JB, Cheng CY, Shu XO, He J, Zheng W, Wong TY, Huang W, Kim BJ, Tai ES, Mohlke KL, Sim $\mathrm{X}$. Association analyses of East Asian individuals and trans-ancestry analyses with European individuals reveal new loci associated with cholesterol and triglyceride levels. Hum Mol Genet. 2017 May 1;26(9):1770-1784. doi: 10.1093/hmg/ddx062. Erratum in: Hum Mol Genet. 2018 Mar 15;27(6):1122. PMID: 28334899; PMCID: PMC6075203.

20. Purcell S, Neale B, Todd-Brown K, et al. PLINK: a tool set for whole-genome association and population-based linkage analyses. Am J Hum Genet. 2007;81(3):559-575.

doi: $10.1086 / 519795$

*Kryzwinski, 2012 http://mkweb.bcgsc.ca/biovis2012/

${ }^{\dagger}$ Mikhailov, 2019 https://ai.googleblog.com/2019/08/turbo-improved-rainbow-colormap-for.html 
NIH $)$ National Library of Medicine

(C) covid-19 Information

Covid-19 Information
Public health information (
Spracklen et al Lipids: Beta $>=0$

$$
\text { - hol - lol - to - ta }
$$

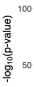

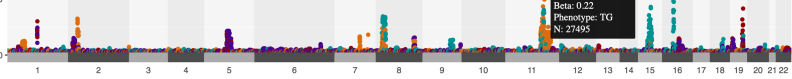

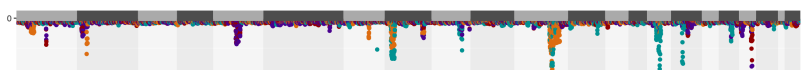

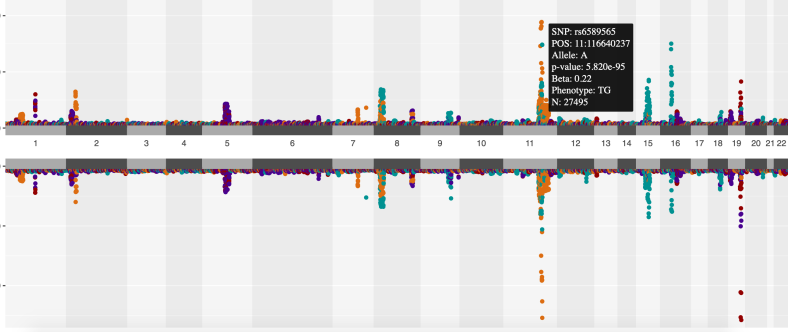

dbSNP

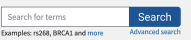

(i) Welcome to the Reference SNP (rs) Report changes. HGVS names are in the HGVS tab. Reference SNP (rs) Report

\section{Switch to classic site \\ rs6589565}

DDownload $\mathbf{A f}$ 口

Organism Homosapiens

Position chr11:116769521 (GRCh38.p13)

Alleles

chr11:116769521 $A>C$ C $A>G / A>T$

Variation Type SNV Single Nucleoside Variation $\mathbf{x}$ 